\title{
A New Approach for Kuhn-Tucker Conditions to Solve Quadratic Programming Problems with Linear Inequality Constraints
}

\author{
Ayansola Olufemi Aderemi ${ }^{1,}$, , Adejumo Adebowale Olusola ${ }^{2}$ \\ ${ }^{1}$ Department of Mathematics \& Statistics, The Polytechnic Ibadan, Ibadan, Nigeria \\ ${ }^{2}$ Department of Statistics, University of Ilorin, Ilorin, Nigeria
}

Email address:

femiayansola@gmail.com (A. O. Aderemi)

${ }^{*}$ Corresponding author

\section{To cite this article:}

Ayansola Olufemi Aderemi, Adejumo Adebowale Olusola. A New Approach for Kuhn-Tucker Conditions to Solve Quadratic Programming Problems with Linear Inequality Constraints. Mathematics and Computer Science. Vol. 5, No. 5, 2020, pp. 86-92.

doi: $10.11648 /$ j.mcs.20200505.11

Received: May 27, 2020; Accepted: September 18, 2020; Published: December 11, 2020

\begin{abstract}
Many real-life problems, such as economic, industrial, engineering to mention but a few has been dealt with, using linear programming that assumes linearity in the objective function and constraint functions. It is noteworthy that there are many situations where the objective function and / or some or all of the constraints are non-linear functions. It is observed that many researchers have laboured so much at finding general solution approach to Non-linear programming problems but all to no avail. Of the prominent methods of solution of Non-linear programming problems: Karush- Kuhn-Tucker conditions method and Wolf modified simplex method. The Karush-Kuhn-Tucker theorem gives necessary and sufficient conditions for the existence of an optimal solution to non-linear programming problems, a finite-dimensional optimization problem where the variables have to fulfill some inequality constraints while Wolf in addition to Karush- Kuhn-Tucker conditions, modified the simplex method after changing quadratic linear function in the objective function to linear function. In this paper, an alternative method for Karush-Kuhn-Tucker conditional method is proposed. This method is simpler than the two methods considered to solve quadratic programming problems of maximizing quadratic objective function subject to a set of linear inequality constraints. This is established because of its computational efforts.
\end{abstract}

Keywords: Karush- Kuhn-Tucker Conditions, New Approach, Quadratic Programming, Wolf Modified Simplex Method

\section{Introduction}

Many real-life problems, such as economic, industrial, engineering to mention but a few has been dealt with, using linear programming that assumes linearity in the objective function and constraint functions. It is noteworthy that there are many situations where the objective function and / or some or all of the constraints are non-linear functions. For instance, to solve a problem in a manufacturing company; as production cost decreases; the production level increases; it requires formulation of the problem into non-linear form. In addition, in cases of large production in the product mix problems; the price of a product depends on the quantity demanded as the more the volume of sales; the lesser the price per unit. These situations also call for quadratic or non-linear programming formulation.

A non-linear programming or quadratic programming problem is a programming problem in which either the objective function or the constraints functions or both are non-linear in decision variables, $\mathrm{X}$.

Kuhn and Tucker [1] proved Kuhn-Tucker theorem and introduced the theory of non-linear programming. It should be noted that Kuhn-Tucker were not the first scholars to prove the theorems as Karush [2] had earlier proven the theorem in his unpublished master's thesis at the University of Chicago. It was on this reason that today; one often sees the theorem referred to as the Karush-Kuhn-Tucker theorem to acknowledge the work of Karush. The Karush-Kuhn-Tucker theorem gives necessary and sufficient conditions for the existence of an optimal solution to 
non-linear programming problems, a finite-dimensional optimization problem where the variables have to fulfill some inequality constraints.

Wolfe Philip [3] introduced a method for solving quadratic programming problems by modifying the simplex method to solve quadratic programming problems using in addition to it; Karush-Kuhn-Tucker conditions and changing the quadratic objective function into a linear objective function.

Allran and Johnsen [4] introduced an algorithm of the penalty function class which solves linear or non-linear optimization problems subject to equality and/or inequality constraints. The algorithm had found considerable application in its ability to distinguish readily between feasible and non-feasible problems.

Terlaky [5] proposed an algorithm which does not require the enlargement of the basic table as Frank-Wolfe [6] method. Terlaky's algorithm is active set method, start from a primal feasible solution to construct dual feasible solution which is complimentary to the primal feasible solution.

Pawar and Ghadle [7] established that an algorithm used to solve one non-linear programming problem, unlike linear programming algorithm performs very well on one type of problem may perform poorly on another problem with a different structure. It is on this basis that a number of algorithm have, in the past, been developed by the researchers, each applicable to a specific type of non-linear programming problem only.

Few other proposed algorithms to solve nonlinear programming problems includes, Zoutendijk [8], Rosen [9], Fiacco and McCormick [10], Li [11], Tang and Wang [12], Iyengar [13], Nasseri, [14], Song et al. [15], Alli and Amir [16], Nayak and Sanjaya [17].

In this study, a proposed algorithm and two of the existing methods vis-a-viz: Kuhn-Tucker condition and simplex like pivoting procedures also called Wolf modified simplex method were employed to solve numerically a maximization problem of quadratic programming type if the objective function is non-linear and with linear inequality constraints.

\section{Materials and Methods}

\subsection{Model Specification}

A general non-linear programming problem of the maximization type is as stated as follows:

$$
\operatorname{MaX} Z=f\left(X_{1}, X_{2}, \cdots, X_{n}\right)
$$

Subject to $h^{i}(X) \leq b_{i}$

$$
X \geq 0, \quad i=1,2, \cdots, m \text {. }
$$

Description of a proposed algorithm

(a) Define the auxiliary (Lagrange) function as

$$
L=f(X)-\sum_{i=1}^{m} \lambda_{i} h^{i}(X)
$$

(b) Differentiate $\mathrm{L}$ with respect to each decision variables $X_{j}$

$$
\frac{\partial L}{\partial x_{i}}=\frac{\partial f(X)}{\partial x_{j}}-\sum_{j=1}^{m} \lambda_{i}\left[\frac{\partial h^{i}(X)}{\partial x_{j}}\right]=0
$$

(c) Consider the following cases, assuming $x_{1}$ and $x_{2}$.

(1) $\lambda_{1}=0, \quad \lambda_{2} \neq 0$

(2) $\lambda_{1} \neq 0, \quad \lambda_{2}=0$.

Procedure for obtaining Kuhn-Tucker conditions

(i) Construct Lagrange function as

$L\left(X_{1} S_{1} \lambda\right)=f(X)-\sum_{i=1}^{m} \lambda_{i}\left[h^{i}(X)+S_{i}^{2}\right]$

(ii) The necessary conditions for minimization are:

(a) $\frac{\partial L}{\partial x_{i}}=\frac{\partial f(X)}{\partial x_{j}}-\sum_{j=1}^{m} \lambda_{i}\left[\frac{\partial h^{i}(X)}{\partial x_{j}}\right]=0$

(b) $\frac{\partial L}{\partial \lambda_{i}}=-\left[h^{i}(X)-S_{i}^{2}\right]=0$

(c) $\frac{\partial L}{\partial S_{i}}=-2 S_{i} \lambda_{i}=0$

$i=1,2, \cdots, m$

$j=1,2, \cdots, n$.

(iii) $\lambda_{i} h^{i}(X)=0$

(iv) $h^{i}(X) \leq 0$.

(v) $\lambda_{i} \geq 0$.

\subsection{Solution}

Assume that we have two Lagrange multipliers $\lambda_{1}$ and $\lambda_{2}$, Four solutions corresponding to the following combinations of $\lambda_{i}(i=1,2)$ can be obtained
(1) $\lambda_{1}=0, \quad \lambda_{2}=0$
(2) $\lambda_{1} \neq 0, \quad \lambda_{2}=0$
(3) $\lambda_{1}=0, \quad \lambda_{2} \neq 0$
(4) $\lambda_{1} \neq 0, \quad \lambda_{2} \neq 0$

Wolfe Modified Simplex Method

Wolfe (1959) introduced Wolfe Modified Simplex Method where two phase simplex method are being modified and transforms of quadratic objective function into linear objective function is made necessary to solve non-linear programming problems having satisfy the requirement or conditions of Karush-Kuhn-Tucker theorem. The method comprises of the following iterative steps:

(i) Change or convert the inequality constraints into equations by adding slack variables $S_{i}^{2}$ and $r_{j}^{2}$ into the constraints.

(ii) Form Lagrange function and obtained 'Karush-Kuhn-Tucker Conditions 
(iii) Add artificial variables $A_{j}, j=1,2, \cdots, n$.

(iv) Form the objective function in linear form as follows:

$$
\operatorname{Min} Z=\sum_{j=1}^{n} A_{j}
$$

(v) Apply the modified two-phase method to obtain the optimal solution to Min $\mathrm{Z}$ which also serves as the optimal solution to the original quadratic programming problem.

\subsection{Numerical Example (Solution by Proposed Modified K-K-T)}

$$
\begin{aligned}
& \operatorname{MaX} Z=200 x+500 y-2 x^{2}-3 y^{2} \\
& \text { s.t. } \quad 2 x+y \leq 140 \\
& 2 x+3 y \leq 180 \\
& x \geq 0, y \geq 0 \\
& L=Z-\lambda_{1}(a)-\lambda_{2}(b)=0 \\
& L=200 x+500 y-2 x^{2}-3 y^{2}-\lambda_{1}(2 x+y-140) \\
& -\lambda_{2}(2 x+3 y-180)=0 \\
& \frac{\partial L}{\partial x}=200-4 x-2 \lambda_{1}-2 \lambda_{2}=0 \\
& \Rightarrow \lambda_{1}+\lambda_{2}=100-2 x \\
& \Rightarrow \lambda_{1}+\lambda_{2}=100-2 x \\
& \frac{\partial L}{\partial y}=500-6 y-\lambda_{1}-3 \lambda_{2}=0 \\
& \Rightarrow \lambda_{1}+3 \lambda_{2}=500-6 y
\end{aligned}
$$

Case 1: $\lambda_{1}=0, \quad \lambda_{2} \neq 0$

(2) becomes

$$
\lambda_{2}=100-2 x
$$

(3) becomes

$$
\lambda_{2}=\frac{500}{3}-2 y
$$

Equating (4) and (5), we have

$$
\begin{gathered}
\therefore 100-2 x=\frac{500}{3}-2 y \\
\Rightarrow-6 x+6 y=200 \\
\Rightarrow-3 x+3 y=100 \\
2 x+3 y=180
\end{gathered}
$$

Solving (6) and (7) simultaneously, we have, $x=16, y=148 / 3$.

(satisfying the two constraints)

$$
\therefore Z=\frac{60160}{3} \approx 20,053.33
$$

Case 2: $\lambda_{1} \neq 0, \quad \lambda_{2}=0$

(2) becomes

$$
\lambda_{1}=100-2 x
$$

(3) becomes

$$
\lambda_{1}=500-6 y
$$

Equating (8) and (9), we have

$$
\begin{gathered}
\Rightarrow \begin{array}{c}
100-2 x=500-6 y \\
-2 x+6 y
\end{array}=400 \\
\Rightarrow-x+3 y=200 \\
2 x+y=140
\end{gathered}
$$

Solving (10) and (11) simultaneously, we have $x=\frac{220}{7}, y=\frac{540}{7}$

(Not satisfying the two constraints)

\subsection{Solution by Karush-Kuhn-Tucker}

$\operatorname{MaX} Z=200 x+500 y-2 x^{2}-3 y^{2}$

s.t.

$$
\begin{aligned}
& 2 x+y \leq 140 \\
& 2 x+3 y \leq 180 \\
& x \geq 0, \quad y \geq 0 .
\end{aligned}
$$

$$
\begin{aligned}
& f(X)=200 x+500 y-2 x^{2}-3 y^{2} \\
& h^{1}(x)=2 x+y-140 \\
& h^{2}(x)=2 x+3 y-180
\end{aligned}
$$

\subsection{Applying K-K-T Conditions}

$$
\begin{gathered}
L=200 x+500 y-2 x^{2}-3 y^{2}-\lambda_{1}(2 x+y-140) \\
-\lambda_{2}(2 x+3 y-180)=0 \\
\lambda_{1}(2 x+y-140)=0 \\
\lambda_{2}(2 x+3 y-180)=0 \\
2 x+y-140 \leq 0 \\
2 x+3 y-180 \leq 0
\end{gathered}
$$




$$
\begin{gathered}
\lambda_{1} \geq 0, \lambda_{2} \geq 0 \\
\frac{\partial L}{\partial x}=200-4 x-2 \lambda_{1}-2 \lambda_{2}=0 \\
\frac{\partial L}{\partial y}=500-6 y-\lambda_{1}-3 \lambda_{2}=0
\end{gathered}
$$

CASE 1: $\lambda_{1}=0, \lambda_{2}=0$.

$\therefore$ (17) becomes $x=50$

(18) becomes $y=\frac{250}{3}$

(15) becomes $\$ 0$

(16) becomes $\$ 0$

It is an infeasible solution.

CASE 2: $\lambda_{1}=0, \quad \lambda_{2} \neq 0$

(17) becomes $\lambda_{2}=100-2 x$

(18) becomes $\lambda_{2}=\frac{500}{3}-2 y$

Equating the result from (17) and (18), we have,

$$
-6 x+6 y=200
$$

Solving (19) and (14), simultaneously, we have,

$$
x=16, \quad y=\frac{148}{3} \text {. }
$$

(15) becomes $<0$

(16) becomes $=0$

It is a feasible solution, therefore $Z=\frac{60160}{3} \approx 20,053.33$

CASE $3: \lambda_{1} \neq 0, \quad \lambda_{2}=0$

(17) becomes $\lambda_{1}=100-2 x$

(18) becomes $\lambda_{1}=500-6 y$

Equating the result from (6) and (7), we have,

$$
x-3 y=-200
$$

Solving (20) and (13), we have

$x=\frac{220}{7}, \quad y=\frac{540}{7}$

(15) becomes $=0$

(16) becomes $\$ 0$

It is an infeasible solution.

CASE $4: \lambda_{1} \neq 0, \quad \lambda_{2} \neq 0$.

Solving (13) and (14) simultaneously, we have, $x=60, \quad y=20$

(15) becomes $=0$

(16) becomes $=0$

It is a feasible solution, therefore, $Z=13,600$

The problem is a maximization type, therefore,

$Z_{\text {max }}=20,053.33$

\subsection{Solution by Wolfe Modified Simplex Method}

$$
\begin{aligned}
& \operatorname{MaX} Z=200 x+500 y-2 x^{2}-3 y^{2} \\
& \text { s.t. } \quad 2 x+y \leq 140 \\
& 2 x+3 y \leq 180 \\
& x, y \geq 0 \text {. } \\
& \operatorname{MaX} Z=200 x+500 y-2 x^{2}-3 y^{2} \\
& \text { s.t. } 2 x+y+s_{1}^{2}=140 \\
& 2 x+3 y+s_{2}^{2}=180 \\
& -x+r_{1}^{2} \quad=0 \\
& -y+r_{2}^{2} \quad=0 \\
& L=200 z+500 y-2 x^{2}-3 y^{2}-\lambda_{1}\left(2 x+y+s_{1}^{2}-140\right) \\
& -\lambda_{2}\left(2 x+3 y+s_{2}^{2}-180\right)-\mu_{1}\left(-x+r_{1}^{2}\right)-\mu_{2}\left(-y+r_{2}^{2}\right) \\
& \frac{\partial L}{\partial x}=200-4 x-2 \lambda_{1}-2 \lambda_{2}+\mu_{1}
\end{aligned}
$$

Setting $\frac{\partial L}{\partial y}=0$

$$
\therefore 4 x+2 \lambda_{1}+2 \lambda_{2}-\mu_{1}=200
$$

$$
\frac{\partial L}{\partial y}=500-6 y-\lambda_{1}-3 \lambda_{2}+\mu_{2}
$$

Setting $\frac{\partial L}{\partial y}=0$

$$
\begin{gathered}
\therefore 6 y+\lambda_{1}+3 \lambda_{2}-\mu_{2}=500 \\
\frac{\partial L}{\partial S_{1}}=-2 \lambda_{1} S_{1}
\end{gathered}
$$

Setting $\frac{\partial L}{\partial S_{1}}=0$

$$
\therefore \quad \lambda_{1} S_{1}=0
$$

$$
\begin{aligned}
& \frac{\partial L}{\partial S_{2}}=-2 \lambda_{2} S_{2} \\
& \frac{\partial L}{\partial S_{2}}=0
\end{aligned}
$$

$$
\therefore \quad \lambda_{2} S_{2}=0
$$

$$
\frac{\partial L}{\partial r_{1}}=-2 \mu_{1} r_{1}
$$

$$
\begin{gathered}
\frac{\partial L}{\partial r_{1}}=0 \\
\therefore \quad \mu_{1} r_{1}=0
\end{gathered}
$$




$$
\begin{aligned}
& \frac{\partial L}{\partial r_{2}}=-2 \mu_{2} r_{2} \\
& \frac{\partial L}{\partial r_{2}}=0 \\
& \therefore \mu_{2} r_{2}=0 \\
& \frac{\partial L}{\partial \lambda_{1}}=-\left(2 x+y+S_{1}^{2}-140\right) \\
& \frac{\partial L}{\partial \lambda_{1}}=0 \\
& \therefore 2 x+y+S_{1}^{2}=140 \\
& \frac{\partial L}{\partial \lambda_{2}}=-\left(2 x+3 y+S_{2}^{2}-180\right) \\
& \frac{\partial L}{\partial \lambda_{2}}=0 \\
& \therefore \quad 2 x+3 y+S_{2}^{2}=180 \\
& \frac{\partial L}{\partial \mu_{1}}=-\left(-x+r_{1}^{2}\right) \\
& \frac{\partial L}{\partial \mu_{1}}=0 \\
& \therefore x=r_{1}^{2} \\
& \Rightarrow \mu_{1} x=\mu_{1} r_{1}^{2} \\
& \frac{\partial L}{\partial \mu_{2}}=-\left(-y+r_{2}^{2}\right) \\
& \frac{\partial L}{\partial \mu_{2}}=0 \\
& \therefore y=r_{2}^{2}
\end{aligned}
$$

\begin{tabular}{|c|c|c|c|c|c|c|c|c|c|c|c|c|}
\hline$C_{j}$ & $\mathbf{0}$ & $\mathbf{0}$ & $\mathbf{0}$ & $\mathbf{0}$ & $\mathbf{0}$ & $\mathbf{0}$ & $\mathbf{0}$ & $\mathbf{0}$ & $\mathbf{0}$ & $\mathbf{0}$ & & \\
\hline Basis & $x$ & $\mathbf{y}$ & $S_{1}$ & $S_{2}$ & $\lambda_{1}$ & $\lambda_{2}$ & $\mu_{1}$ & $\mu_{2}$ & $A_{1}$ & $A_{2}$ & $b_{i}$ & $b_{i} / y$ \\
\hline$R_{1} \rightarrow A_{1}: 1$ & 4 & 0 & 0 & 0 & 2 & 2 & -1 & 0 & 1 & 0 & 200 & - \\
\hline$R 2 \rightarrow A_{2}: 1$ & 0 & 6 & 0 & 0 & 1 & 3 & 0 & -1 & 0 & 1 & 500 & 83.33 \\
\hline$R_{3} \rightarrow S_{1}: 0$ & 2 & 1 & 1 & 0 & 0 & 0 & 0 & 0 & 0 & 0 & 140 & 140 \\
\hline$R_{4} \rightarrow S_{2}: 0$ & 2 & 3 & 0 & 1 & 0 & 0 & 0 & 0 & 0 & 0 & 180 & 60 \\
\hline$Z_{j}$ & 4 & 6 & 0 & 0 & 3 & 5 & -1 & -1 & 1 & 1 & & \\
\hline$C_{j}-Z_{j}$ & -4 & -6 & 0 & 0 & -3 & -5 & 1 & 1 & 0 & 0 & & \\
\hline
\end{tabular}

$$
\left.\begin{array}{c}
\Rightarrow \mu_{2} y=\mu_{2} r_{2}^{2} \\
\lambda_{1} S_{1}=\lambda_{2} S_{2}=0 \\
\mu_{1} x=\mu_{2} y=0
\end{array}\right]
$$

$$
\operatorname{Min} Z=A_{1}+A_{2}
$$

$$
\begin{array}{ll}
\text { s.t. } & 4 x+2 \lambda_{1}+2 \lambda_{2}-\mu_{1}+A_{1}=200 \\
& 6 y+\lambda_{1}+3 \lambda_{2}-\mu_{2}+A_{2}=500 \\
& 2 x+y+S_{1} \quad=140 \\
& 2 x+3 y+S_{2} \quad= \\
& x, y, S_{1}, S_{2}, \lambda_{1}, \lambda_{2}, \mu_{1}, \mu_{2}, A_{1} \text { and } A_{2} \geq 0 .
\end{array}
$$

Table 1. Initial Solution.

Table 2. First Iteration.

\begin{tabular}{lcccccccccccc}
\hline \multirow{2}{*}{ Basis } & $\mathbf{0}$ & $\mathbf{0}$ & $\mathbf{0}$ & $\mathbf{0}$ & $\mathbf{0}$ & $\mathbf{0}$ & $\mathbf{0}$ & $\mathbf{0}$ & $\mathbf{1}$ & $\mathbf{1}$ & \\
\cline { 2 - 11 } & $\boldsymbol{x}$ & $\mathbf{Y}$ & $\boldsymbol{S}_{1}$ & $\boldsymbol{S}_{2}$ & $\boldsymbol{\lambda}_{1}$ & $\boldsymbol{\lambda}_{2}$ & $\boldsymbol{\mu}_{1}$ & $\boldsymbol{\mu}_{2}$ & $\boldsymbol{A}_{1}$ & $\boldsymbol{A}_{2}$ & $\boldsymbol{b}_{\boldsymbol{i}}$ & $\boldsymbol{b}_{\boldsymbol{i}} / \boldsymbol{x}$ \\
\hline$R_{1}^{1}=R_{1} \rightarrow A_{1}: 1$ & 4 & 0 & 0 & 0 & 2 & 2 & -1 & 0 & 1 & 0 & 200 & 50 \\
$R_{2}^{1}=R_{2}-6 R_{4}^{1} \rightarrow A_{2}: 1$ & -4 & 0 & 0 & -2 & 1 & 3 & 0 & -1 & 0 & 1 & 140 & - \\
\hline
\end{tabular}




\begin{tabular}{|c|c|c|c|c|c|c|c|c|c|c|c|c|}
\hline \multirow[b]{2}{*}{ Basis } & $\mathbf{0}$ & $\mathbf{0}$ & $\mathbf{0}$ & $\mathbf{0}$ & $\mathbf{0}$ & $\mathbf{0}$ & $\mathbf{0}$ & $\mathbf{0}$ & 1 & 1 & & \\
\hline & $x$ & $\mathbf{Y}$ & $S_{1}$ & $\boldsymbol{S}_{2}$ & $\lambda_{1}$ & $\lambda_{2}$ & $\mu_{1}$ & $\mu_{2}$ & $A_{1}$ & $A_{2}$ & $b_{i}$ & $b_{i} / x$ \\
\hline$R_{3}^{1}=R_{3}-R_{4}^{1} \rightarrow S_{1}: 0$ & $\frac{4}{3}$ & 0 & 1 & $\frac{-1}{3}$ & 0 & 0 & 0 & 0 & 0 & 0 & 80 & 60 \\
\hline$R_{4}^{1}=\frac{R_{4}}{3} \rightarrow y: 0$ & $\frac{2}{3}$ & 1 & 0 & $\frac{1}{3}$ & 0 & 0 & 0 & 0 & 0 & 0 & 60 & 90 \\
\hline$Z_{j}$ & 0 & 0 & 0 & -2 & 3 & 5 & -1 & -1 & 1 & 1 & & \\
\hline$C_{j}-Z_{j}$ & 0 & 0 & 0 & 2 & -3 & -5 & 1 & 1 & 0 & 0 & & \\
\hline
\end{tabular}

Table 3. Second Iteration

\begin{tabular}{|c|c|c|c|c|c|c|c|c|c|c|c|}
\hline$C_{j}$ & 0 & 0 & 0 & 0 & 0 & 0 & 0 & 0 & 1 & & \\
\hline Basis & $x$ & $\mathbf{Y}$ & $S_{1}$ & $S_{2}$ & $\lambda_{1}$ & $\lambda_{2}$ & $\mu_{1}$ & $\mu_{2}$ & $A_{2}$ & $b_{i}$ & $b_{i} / x$ \\
\hline$R_{1}^{11}=\frac{R_{1}^{1}}{4} \rightarrow x: 0$ & 1 & 0 & 0 & 0 & $\frac{1}{2}$ & $\frac{1}{2}$ & $\frac{-1}{4}$ & 0 & 0 & 50 & 100 \\
\hline$R_{2}^{11}=R_{2}^{1}+4 R_{1}^{11} \rightarrow A_{2}: 1$ & 0 & 0 & 0 & -2 & 3 & 5 & -1 & -1 & 1 & 340 & 68 \\
\hline$R_{3}^{11}=R_{3}^{1}-\frac{4}{3} R_{1}^{11} \rightarrow S_{1}: 0$ & 0 & 0 & 1 & $\frac{-1}{3}$ & $\frac{-2}{3}$ & $\frac{-2}{3}$ & $\frac{1}{3}$ & 0 & 0 & $\frac{40}{3}$ & - \\
\hline$R_{4}^{11}=R_{4}^{1}-\frac{2}{3} R_{1}^{11} \rightarrow y: 0$ & 0 & 1 & 0 & $\frac{1}{3}$ & $\frac{-1}{3}$ & $\frac{-1}{3}$ & $\frac{1}{6}$ & 0 & 0 & $\frac{80}{3}$ & - \\
\hline$Z_{j}$ & 0 & 0 & 0 & -2 & 3 & 5 & -1 & -1 & 1 & & \\
\hline$C_{j}-Z_{j}$ & 0 & 0 & 0 & 2 & -3 & -5 & 1 & 1 & 0 & & \\
\hline
\end{tabular}

Table 4. Final Solution.

\begin{tabular}{llllllllll}
\hline $\boldsymbol{C}_{\boldsymbol{j}}$ & $\mathbf{0}$ & $\mathbf{0}$ & $\mathbf{0}$ & $\mathbf{0}$ & $\mathbf{0}$ & $\mathbf{0}$ & $\mathbf{0}$ & $\mathbf{0}$ & \\
\hline Basis & $\boldsymbol{x}$ & $\mathbf{Y}$ & $\boldsymbol{S}_{1}$ & $\boldsymbol{S}_{2}$ & $\boldsymbol{\lambda}_{1}$ & $\lambda_{2}$ & $\boldsymbol{\mu}_{1}$ & $\boldsymbol{\mu}_{2}$ & $\boldsymbol{b}_{\boldsymbol{i}}$ \\
\hline$R_{1}^{111}=R_{1}^{11}-\frac{1}{2} R_{2}^{111} \rightarrow x: 0$ & 1 & 0 & 0 & $\frac{1}{5}$ & $\frac{1}{5}$ & 0 & $\frac{-3}{20}$ & $\frac{1}{10}$ & 16 \\
$R_{2}^{111}=\frac{R_{2}^{11}}{5} \rightarrow \lambda_{2}: 0$ & 0 & 0 & 0 & $\frac{-2}{5}$ & $\frac{3}{5}$ & 1 & $\frac{-1}{5}$ & $\frac{-1}{5}$ & 68 \\
$R_{3}^{111}=R_{3}^{11}+\frac{2}{3} R_{2}^{111} \rightarrow S_{1}: 0$ & 0 & 0 & 1 & $\frac{-49}{15}$ & $\frac{-4}{15}$ & 0 & $\frac{1}{5}$ & $\frac{-2}{15}$ & $\frac{176}{3}$ \\
$R_{4}^{111}=R_{4}^{11}+\frac{1}{3} R_{2}^{111} \rightarrow y: 0$ & 0 & 1 & 0 & $\frac{1}{5}$ & $\frac{-2}{15}$ & 0 & $\frac{1}{10}$ & $\frac{-1}{15}$ & $\frac{148}{3}$ \\
$Z_{j}$ & 0 & 0 & 0 & 0 & 0 & 0 & 0 & 0 & \\
$C_{j}-Z_{j}$ & 0 & 0 & 0 & 0 & 0 & 0 & 0 & 0 & \\
\hline
\end{tabular}

$$
\begin{aligned}
x=16, y=\frac{148}{3}, Z_{\text {MaX }} & =200(16)+500\left(\frac{148}{3}\right)-2(16)^{2}-3\left(\frac{148}{3}\right)^{2} \\
& =3200+\frac{74000}{3}-512-\frac{21904}{3} \\
& =\frac{60160}{3}
\end{aligned}
$$$$
=20053.33 \mathrm{~K}
$$

\section{Discussion of Results}

The two existing methods of solution to solving Non-linear programming problems considered in this study; viz-a-vis; Karush-Kuhn-Tucker and Wolf Modified Simplex Methods proven to be consistent and efficient for the proposed method gives the same optimal results of the maximization problems. i.e $Z_{\max }=20053.33 \mathrm{~K}, \mathrm{x}=16, y=148 / 3$

The numerical results of our proposed method satisfied the Kuhn-Tucker conditions and gives optimum solution as other methods did. However, an efficient method for the solution of general non-linear programming problem is still an area of future research. 


\section{Conclusion}

Non- linear Programming came into being due to the fact that not all problems are in linear functional forms.

There are many methods of solution for solving different type of Non-linear Problems but this study search and found an alternative method for Kuhn-Tucker conditions method to obtain the solution of quadratic programming problems has been proposed. This technique is useful to apply on numerical problems for it reduces the labour work and save valuable time. The technique is very effective like Karush-Kuhn-Tucker conditions and Wolf Modified Simplex Method but it is simpler than the two methods because of its computational efforts.

\section{References}

[1] Kuhn, H. W., and Tucker, A. W. (1950) Non-linear Programming In Proceedings of the Second Berkeley Symposium on Mathematical Statistics and Probability, J. Neyman, Ed., pp. 481-492, Berkeley.

[2] Karush, W. (1939) Minima of Functions of Several Variables with Inequalities as Side Conditions. Dissertation, Department of Mathematics, University of Chicago.

[3] Wolfe Philip (1959) The Simplex Method for Quadratic Programming, The Econometric Society, Econometrica, Vol. 27, pp. 382-398.

[4] Allran, R. R and Johnsen, S. E. J (1970) “An Algorithm for Solving Non-linear programming problems subject to non-linear inequality constraints" The computer Journal, Vol. 13, No 2. Pp 171-177.

[5] Terlaky T. A. (1987) New Algorithm for Quadratic Programming, European Journal of Operation Research, Vol. 32, pp. 294-301, North-Holland.

[6] Frank M. and Wolfe, P. (1956) An Algorithm for Quadratic Programming, Naval Research Logistics Quarterly March-June, pp. 95-110.
[7] Pawar, T. S and Ghadle, K. P. (2015) "New approach for Wolfe's modified simplex method to solve Quadratic programming problems" International Journal of Research in Engineering and Technology, vol. 04, issue 01, pp. 371-376.

[8] Zoufendijk, G. (1960) Methods of Feasible Directions. Amsterdem and New York: Elsevier Publishing Company.

[9] Rosen, J. B (1961) The Gradient Project Method for Nonlinear Programming. Part II: Nonlinear Constraints, Shall Development Company, Emeryville, California.

[10] Fiacco, A. V. and McCormick, G. P. (1963) Programming under Nonlinear Constraints by Unconstrained Minimization: A Primal-Dual Method, Research Analysis Corporation, McLean, Virginia, Technical Paper, RAC-TP-96.

[11] Li, J. X. (1994). On an Algorithm for Solving Fuzzy Linear Systems, Fuzzy Sets and Systems 61, 369-371.

[12] Tang, J. and Wang, D. (1996) Modelling and Optimization for a type of Fuzzy Nonlinear Programming Problems in Manufacturing Systems, Proceedings of the 35th Conference on Decision and Control, Kobe, Japan.

[13] Iyengar, P. (2002) "Non-Linear Programming; Introduction", IEOR, Handout 19, 16 October.

[14] Song, Y. Chen, Y. and Wu, X. (2005) "A Method for Solving Nonlinear Programming Models with All Fuzzy Coefficients Based on Genetic Algorithm", Advances in Natural Computation, Vol. 36, No. 11, pp, 1101-1104.

[15] Nasseri, S. H. (2008) "Fuzzy Nonlinear Optimization, "The Journal of Nonlinear Analysis and its Applications, Vol. 1, No. 4, pp. $230-235$.

[16] Ali, F. J. and Amir, S. (2012). "Solving Nonlinear Programming Problem in Fuzzy Environment", Int. J. Contemp. Math. Sciences, Vol. 7, No. 4, pp. 159-170.

[17] Nayak, J. and Sanjaya, K. B. (2012). "Optimal Solution of Fuzzy Nonlinear Programming Problems with Linear Constraints", International Journal of Advances in Science and Technology, Vol. 4, No. 4, pp. 43-52. 